\title{
Prevalencia de caries en escolares de 6 a 15 años, Isla de Pascua
}

\section{Caries prevalence in 6 to 15 year-old school children, from Easter Island}

\author{
Gómez González V¹, Cabello Ibacache R ${ }^{1}$, Rodríguez Martínez G ${ }^{1}$, Urzúa Araya I', Silva Steffens N², \\ Phillips Letelier $\mathrm{M}^{3}$, Yévenes López $\mathrm{I}^{4}$
}

\begin{abstract}
RESUMEN
Objetivo: El objetivo de este estudio fue determinar la prevalencia y severidad de caries de piezas permanentes y algunos factores de riesgo relacionados en un grupo de escolares de Isla de Pascua. Materiales y Métodos: 116 niños de 6 a 15 años de edad fueron seleccionados al azar. Se efectuó examen clínico individualizado y se registraron los índices COP-D, significante de caries (SIC), de Higiene Oral de Greene y Vermillon (G-V) y se realizó una encuesta de dieta. Para conocer la frecuencia y recuento de Streptococcus mutans y Streptococcus sobrinus, se tomaron muestras de saliva estimulada a 23 niños Rapa Nui elegidos al azar. Finalmente, se analizaron muestras de agua potable en 3 sitios distintos de la isla para medir la cantidad de flúor disponible. Resultados: La prevalencia de caries en piezas permanentes fue 38\%. El COP-D fue 1.19. El valor promedio del G-V fue 1.63. Se aisló e identificó $S$. mutans y $S$. sobrinus en el $56.2 \%$ y $13.2 \%$ respectivamente. El recuento promedio para $S$. mutans fue de $3.3 \times 105$ UFC/ml saliva y para S. sobrinus fue de 7.2x104 UFC/ml. Se observó un promedio de 0.223 ppm/Fluoruro en el agua. Desviación Estándar 0.025 ppm. Según la encuesta de dieta el $30 \%$ de los niños presenta bajo riesgo de caries, el $47 \%$ presenta mediano riesgo y el $23 \%$ presenta alto riesgo. Conclusión: El 38\% presenta historia de caries en piezas permanentes. El COP-D promedio es 1.19. En niños Rapa Nui 0.9 y en No Rapa Nui 1.77 . Rev. Clin. Periodoncia Implantol. Rehabil. Oral Vol. 5(2); 69-73, 2012.
\end{abstract}

Palabras clave: Epidemiología, caries dental, factores de riesgo.

\section{ABSTRACT}

Aim: The aim of this study was to determine the prevalence and severity of caries and some related risk factors in a group of school children from Easter Island. Materials and Methods: 116 children aged 6 to 15 years were randomly selected. Individual clinical examination was performed and index COP-D, significant caries index (SIC), Greene and Vermillon Oral Hygiene index (GV) were recorded. A diet survey was performed. To determine the frequency and count of Streptococcus mutans and Streptococcus sobrinus, stimulated saliva samples were collected from 23 Rapa Nui children randomly selected. Finally, samples of drinking water in 3 different places of the island were analyzed to measure the amount of fluoride available. Results: Caries prevalence was $38 \%$. COP-D index was 1.19. The mean value of G-V was 1.63. S. mutans and S. sobrinus were isolated and identified in $56.2 \%$ and $13.2 \%$ of the samples, respectively. The average count for S. mutans was $3.3 \times 105 \mathrm{CFU} / \mathrm{ml}$ saliva and for S. sobrinus was $7.2 \times 104 \mathrm{CFU} /$ $\mathrm{ml}$. A mean of 0.223 Standard Deviation $0.025 \mathrm{ppm} /$ Fluoride was observed in drinking water. According to the diet survey, $30 \%$ of children have a low risk of caries, $47 \%$ have medium risk and $23 \%$ a high risk. Conclusion: According to this study $38 \%$ have caries history in permanent teeth. Mean COP-D index was 1.19. In Rapa Nui children 0.9 and Not Rapa Nui children 1.77.

Rev. Clin. Periodoncia Implantol. Rehabil. Oral Vol. 5(2); 69-73, 2012.

Key words: Epidemiology, dental caries, indicator factors.

\section{INTRODUCCIÓN}

Los estudios epidemiológicos permiten entender el impacto de una determinada enfermedad, tanto en relación a su importancia clínica como desde el punto de vista de la salud pública contribuyendo a la comprensión del proceso, sus factores e indicadores de riesgo y a la determinación de los métodos efectivos para su prevención, tratamiento y mantención ${ }^{(1)}$. Estas investigaciones epidemiológicas son importantes como información de base para generar políticas públicas enfocadas a mejorar la salud de la población a partir de la evidencia disponible ${ }^{(2)}$. Para esto, es fundamental que se recoja información relevante en estudios epidemiológicos apropiados ${ }^{(1)}$.

A pesar de existir una marcada reducción de la prevalencia de caries dental, esta patología sigue siendo el mayor problema de salud oral en la mayoría de los países industrializados, afectando entre el 60 y $90 \%$ de la población escolar y a la gran mayoría de los adultos, siendo especialmente prevalente en los países Latinoamericanos ${ }^{(3,4)}$.
En las recientes décadas en la mayoría de los países desarrollados ha ocurrido una marcada reducción de prevalencia de lesiones de caries ${ }^{(2)}$, principalmente observada en los países nórdicos. Esto se observa en el aumento del porcentaje de pacientes libres de lesiones de caries, en la disminución del índice COP-D y especialmente del componente $C$ de este índice ${ }^{(3)}$. La disminución de este índice ha sido acompañada de un gran cambio en la distribución a través del tiempo de las lesiones en una población dada ${ }^{(5)}$. Las razones por las cuales se ve esta disminución en la prevalencia de caries se atribuyen a la fluoruración del agua potable, al uso masivo de pastas fluoruradas, estándares más conservadores en el criterio de diagnóstico de caries, tratamiento de lesiones cariosas y la aplicación de medidas de control del proceso de caries ${ }^{(5)}$. Otro factor involucrado en la disminución de esta prevalencia ha sido la mejoría de los estándares socioeconómicos de la población en los países desarrollados, así como el énfasis que han puesto en mejorar la salud bucal ${ }^{2,6,7,8)}$.

En Chile los problemas de salud bucodental son de alta prevalencia, al igual que los existentes en otros países en vías de desarrollo ${ }^{(9)}$.

1. Área de Cariología. Departamento de Odontología Restauradora. Facultad de Odontología, Universidad de Chile. Chile.

2. Área de Microbiología. Facultad de Odontología, Universidad de Chile. Chile.

3. Curso Cariología y Operatoria Dental. Facultad de Medicina, Clínica Alemana-Universidad del Desarrollo. Chile.

4. Área de Química. Facultad de Odontología, Universidad de Chile. Chile.

Correspondencia autor: Valeria Carolina Gómez González. valeria.gomez.g@gmail.com. Departamento Odontología Restauradora. Facultad de Odontología, Universidad de Chile. Sergio Livingstone P. 342, Independencia, Chile. Trabajo recibido el 18/10/2011. Aceptado para su publicación el 06/05/2012. 
Las características microbiológicas y clínicas, tanto para caries dental como para enfermedades gingivales, han sido ampliamente estudiadas en poblaciones infantiles en el mundo(1). En el caso de la caries dental los estudios microbiológicos se realizan con el fin de establecer asociaciones con la ocurrencia de Streptococcus mutans y Streptococcus sobrinus de manera que se puedan instaurar medidas preventivas de la patología ${ }^{(1,10)}$. En Chile hay escasa información relacionada a la presencia de estos microorganismos así como de las características clínicas de caries y de problemas gingivales en los grupos étnicos. Uno de estos grupos son los "Rapa Nui", habitantes de Isla de Pascua, la cual está ubicada en el océano Pacífico conformando el vértice más oriental del triángulo de la polinesia. Tiene una superficie de $163,6 \mathrm{~km}^{2}$ (la mayor de las islas del Chile insular) y una población de 4.537 habitantes, proyectada al 2009, según cálculos del Instituto Nacional de Estadísticas (INE), concentrados principalmente en la capital Hanga Roa ${ }^{(11)}$. La Isla de Pascua es parte del territorio nacional y se encuentra aproximadamente a 3.500 kilómetros del continente, lo que determina una condición de aislamiento y de escasez de servicios, especialmente de cuidados de salud. Los servicios de atención odontológica prestados por el gobierno de Chile se concentran en sólo un profesional que debe responder a las necesidades de toda la población de la isla. Por otro lado, la población que habita la región alberga una alta proporción de individuos de origen Rapa Nui, quienes conservan hábitos, costumbres y creencias que los diferencian de los habitantes con otros orígenes. Es por esto que tenemos la necesidad de explorar el comportamiento de la caries dental en este grupo peculiar de individuos. Los hallazgos de este estudio podrán ser utilizados por los planificadores en salud para el desarrollo de políticas públicas dirigidas a condiciones específicas de grupos étnicos como el Rapa Nui. Este estudio tiene como objetivo determinar la prevalencia y severidad de caries dental en dientes permanentes y explorar algunos factores de riesgo de esta enfermedad en un grupo de escolares de 6 a 15 años de edad de Isla de Pascua de Chile, durante el año 2008.

\section{MATERIALES Y MÉTODOS}

\section{Población}

La población de este estudio de corte transversal corresponde a la población escolar de niños y niñas de la comuna de Isla de Pascua, la cual está constituida por 1.137 individuos entre 5 y 19 años, que están distribuidos en cuatro establecimientos educacionales ${ }^{(11)}$. Estos colegios tienen dependencias administrativas de tipo municipal y particular subvencionado.

\section{Muestra}

La muestra se construyó en dos etapas. Primero se seleccionó del total de establecimientos educacionales uno al azar, el Colegio Católico Hermano Eugenio Eyraud, que depende administrativamente de la forma particular subvencionado. En una segunda etapa se seleccionaron individuos que asisten al establecimiento escogido hasta alcanzar el tamaño de muestra determinado. Los criterios de selección incluyen a todos los niños presentes al momento del examen que tenían entre 5 y 19 años de edad. Para su selección se utilizaron números azarosos los que permiten escoger desde las listas de asistencia a quienes fueron finalmente examinados.

El tamaño de la muestra se estimó en 100 individuos considerando una prevalencia de caries de un $70 \%{ }^{(9)}$, con un nivel de confianza del $95 \%$ y un error de estimación de 9 puntos. Se consideró un sobre muestreo del $10 \%$, lo que determinó un tamaño muestral de 110 individuos.

\section{Recolección de la Información}

\section{Examen Bucal}

Luego de obtener el consentimiento informado de las autoridades del colegio y los padres de los sujetos seleccionados, un examinador capacitado y calibrado en la detección de lesiones de caries (Índice Kappa 0.8) realizó el examen clínico en dependencias del colegio utilizando instrumental clínico estéril y luz artificial. En una ficha individualizada, especialmente diseñada para este estudio y sobre las bases de la Organización Mundial de la Salud (OMS), se registró la información recogida utilizando los criterios de detección de lesiones de caries de la $\mathrm{OMS}^{(12)}$. Se obtuvieron datos relacionados con identificación de los individuos, como edad y sexo, además el estado de la dentición permanente para determinar la prevalencia de caries (proporción de individuos con índice COP-D mayor que 0) y el índice COP-D. No se realizó la evaluación del estado de los dientes temporales. Se evaluó el nivel de placa visible de dientes índices para la construcción del índice de Greene y Vermillon (G-V)(13).

Se construyó el índice COP-D que corresponde a la suma de los dientes cariados, obturados y perdidos por caries ${ }^{(12)}$. Una vez obtenido los valores de este índice para la muestra en estudio se efectuó el cálculo del Índice Significante de Caries (SIC)(14), para conocer el promedio del índice COP-D del tercio más afectado de esta población.

Además, se determinó el origen Rapa Nui de los individuos. Para esto se consideró que todos aquellos individuos con apellidos originarios correspondían a la categoría Rapa Nui. Para determinar si un apellido correspondía a un apellido originario se consultó a miembros reconocidos oriundos de la comunidad Rapa Nui.

\section{Muestras de Saliva}

Adicionalmente, sobre una sub-muestra por conveniencia de 23 niños y niñas de origen Rapa Nui, se tomó muestras de saliva estimulada. El criterio empleado para la selección de esta muestra se vincula con la disposición a colaborar en el estudio de los individuos. Las muestras fueron mantenidas en frío para su transporte y posterior procesamiento. En el laboratorio de microbiología de la Facultad de Odontología de la Universidad de Chile se realizaron diluciones seriadas de las muestras de saliva, $100 \mu$ lts de dilución apropiada $\left(10^{-2}\right)$, se sembraron en medio TYCSB y se incubaron a $37^{\circ} \mathrm{C}$ por $48 \mathrm{hrs}$ en condiciones de anaerobiosis. Posteriormente se contaron las colonias adherentes compatibles con S. mutans y $S$. sobrinus. Se realizaron subcultivos de los distintos tipos coloniales para realizar pruebas bioquímicas y extracción de DNA. Se biotipificaron los aislados y el DNA se mantuvo a $-20^{\circ} \mathrm{C}$ hasta su utilización para PCR, según Oho T y cols. ${ }^{(15)}$.

\section{Encuesta de Dieta}

Con la finalidad de conocer el potencial cariogénico y la frecuencia de consumo de alimentos de la muestra en estudio, en una segunda visita al establecimiento educacional, se aplicó la encuesta pre codificada y pre testeada de dieta traducida al español desde el instrumento original de Holm y cols. ${ }^{(16)}$ a una sub-muestra por conveniencia de 30 alumnos, quienes fueron seleccionados de acuerdo a la disponibilidad de responder el cuestionario. Este instrumento no se encuentra validado en el contexto internacional, sin embargo es recomendado su uso por expertos en el área ${ }^{(2)}$. En primer lugar, se determinó el promedio del contenido de carbohidratos por 100 $\mathrm{mg}$ o $\mathrm{ml}$ correspondiente para cada alimento incluido en la encuesta de dieta, basados en la información nutricional entregada por el fabricante. Posteriormente se clasificó cada alimento según consistencia, siendo estas No pegajoso y Altamente pegajoso según el criterio de un grupo de expertos.

Finalmente, el potencial cariogénico de la dieta consumida se obtuvo multiplicando las variables: frecuencia diaria de consumo, contenido de carbohidrato y consistencia de cada alimento, lo que entrega un número ordinal que fue promediado entre todos los alimentos considerados en la encuesta. Las variables ordinales se ordenaron de menor a mayor y luego se dividieron en tercios. Cada tercio fue clasificado en la condición de alto, mediano o bajo riesgo, respectivamente, de acuerdo a la posición de los valores en esta división.

\section{Muestras de Agua Potable}

Para determinar el nivel de fluoruro en agua potable de consumo se tomaron muestras en tres sitios distintos de la isla. La determinación de fluoruro, se realizó en forma potenciométrica utilizando un electrodo específico OAKTON y un analizador de iones OAKTON Ion 510 Series, de acuerdo a la técnica modificada descrita por Yévenes y cols. ${ }^{(17,18)}$. A 5 $\mathrm{ml}$ de agua filtrada se agregó $5 \mathrm{ml}$ de TISAB II y se leyó directamente en el ionómetro, previamente calibrado, obteniéndose lecturas en partes por millón (ppm) de fluoruro.

\section{Análisis de Datos}

Se determinó la prevalencia de caries utilizando la proporción 
de individuos libre de lesiones caries en la dentición permanente, los índices COP-D, SIC y G-V de acuerdo a lo reportado por los autores ${ }^{(12)}$, y se realizaron estimaciones utilizando intervalos de confianza del $95 \%$ para cada uno de estos parámetros. Se estableció una aproximación a los niveles de $S$. mutans y $S$. sobrinus mediante la cuantificación de Unidades Formadoras de Colonia (UFC) en muestras de saliva. Se estableció el potencial cariogénico de la dieta consumida y la concentración en ppm del ión fluoruros en el agua potable. Para los análisis estadísticos se calcularon medias y proporciones y se realizaron las estimaciones de los parámetros correspondientes. Para la ejecución del análisis se utilizó Programa Stata 9. Se presentaran los resultados del análisis de datos en tablas.

\section{RESULTADOS}

La muestra final quedó constituida por 116 individuos entre 6 y 15 años de edad con una media de 10.09 años (IC 95\% 9.6-10.6), lo que representa aproximadamente un $10.2 \%$ del total de la población escolar de la isla. La muestra estuvo constituida por $53 \%$ hombres y $47 \%$ de mujeres. La caracterización de la muestra se presenta en la Tabla 1.

Tabla 1. Características de la muestra.

\begin{tabular}{|c|c|c|c|}
\hline \hline Origen & Sexo & $\mathbf{n}$ & Porcentaje \\
\hline \multirow{2}{*}{ Rapa Nui } & Mujer & 32 & 41.6 \\
\cline { 2 - 4 } & Hombre & 45 & 58.4 \\
\hline \multirow{2}{*}{ Total } & & $\mathbf{7 7}$ & $\mathbf{6 6 . 4}$ \\
\hline \multirow{2}{*}{ No Rapa Nui } & Mujer & 23 & 58.9 \\
\cline { 2 - 4 } & Hombre & 16 & 41.1 \\
\hline Total & & $\mathbf{3 9}$ & $\mathbf{3 3 . 6}$ \\
\hline Total & & $\mathbf{1 1 6}$ & $\mathbf{1 0 0 \%}$ \\
\hline
\end{tabular}

Con respecto a la prevalencia de caries en dentición permanente, el 38.8\% (IC 95\% 29.9-47.8) de los niños tiene historia de caries, por lo tanto el $61.2 \%$ (IC 95\% 52.2-70.2) está libre de lesiones de caries en dientes permanentes. La media del índice COP-D es de 1.19 (IC 95\% 0.8-1.6) con un rango entre 0 y 8 (DE 2.06). Los resultados del índice COP-D estratificados por sexo y origen se presentan en la Tabla 2.

Tabla 2. Promedio del índice COP-D según características de la muestra.

\begin{tabular}{|c|c|c|c|c|}
\hline & $\mathbf{n}$ & $\begin{array}{c}\text { Índice } \\
\text { COP-D }\end{array}$ & IC 95\% & Rango \\
\hline Hombres & 61 & 1.18 & $0.7-1.7$ & $0-8$ \\
\hline Mujeres & 55 & 1.2 & $0.6-1.7$ & $0-8$ \\
\hline Total & $\mathbf{1 1 6}$ & & & \\
\hline Rapa Nui & 77 & 0.9 & $0.5-1.3$ & $0-7$ \\
\hline No Rapa Nui & 39 & 1.77 & $0.9-2.6$ & $0-8$ \\
\hline Total & $\mathbf{1 1 6}$ & & & \\
\hline Total Muestra & $\mathbf{1 1 6}$ & $\mathbf{1 . 1 9}$ & $\mathbf{0 . 8 - 1 . 6}$ & $\mathbf{0 - 8}$ \\
\hline
\end{tabular}

En la Tabla 3 se presenta el índice COP-D por tramo de edad. 2.37-3.98).

El índice significante de caries (SIC) fue de 3.18 (IC 95\%

En relación al valor del G-V promedio para toda la muestra fue
1.63 (IC 95\% 1.53-1.74). En hombres se observó un valor promedio de 1.66 (IC 95\% 1.51-1.81) y en mujeres de 1.60 (IC 95\% 1.45-1.76). Para la muestra de niños Rapa Nui el valor promedio del índice fue 1.69 (IC $95 \% 1.55-1.82$ ) y para la muestra No Rapa Nui 1.53 (IC 95\% 1.36-1.70).

Tabla 3. Índice COP-D por tramo de edad

\begin{tabular}{|c|c|c|c|}
\hline \hline Grupo de edad & $\mathbf{N}$ & COP-D & IC $95 \%$ \\
\hline 6 a 8 años & 38 & 1.24 & $0.41-2.06$ \\
\hline 9 a 12 años & 49 & 0.82 & $0.46-1.18$ \\
\hline 13 a 15 años & 29 & 1.76 & $0.91-2.6$ \\
\hline Total & $\mathbf{1 1 6}$ & $\mathbf{1 . 1 9}$ & $\mathbf{0 . 8 - 1 . 6}$ \\
\hline
\end{tabular}

Tabla 4. Índice de Greene y Vermillon según características de la muestra.

\begin{tabular}{|c|c|c|}
\hline \hline & $\begin{array}{c}\text { Índice de Greene } \\
\text { y Vermillon }\end{array}$ & IC 95\% \\
\hline Hombres & 1.66 & $1.51-1.81$ \\
\hline Mujeres & 1.60 & $1.45-1.76$ \\
\hline Rapa Nui & 1.69 & $1.55-1.82$ \\
\hline No Rapa Nui & 1.53 & $1.36-1.70$ \\
\hline Total Muestra & $\mathbf{1 . 6 3}$ & $\mathbf{1 . 5 3 - 1 . 7 4}$ \\
\hline
\end{tabular}

En las muestras de saliva analizadas se aisló e identificó $S$. mutans y $S$. sobrinus en el $56.2 \%$ y $13.2 \%$, respectivamente. El recuento promedio para S. mutans fue de $3.3 \times 105 \mathrm{UFC} / \mathrm{ml}$ saliva y para $S$. sobrinus fue de $7.2 \times 104$ UFC/ml de saliva.

Con respecto a la encuesta de dieta, el $30 \%$ presenta bajo riesgo de caries. El $47 \%$ presenta mediano riesgo y el $23 \%$ presenta alto riesgo. En relación con el análisis del agua, se observó un promedio de 0.223 (Desviación Estándar 0.025) ppm/Fluoruro.

\section{DISCUSIÓN}

Este el primer estudio efectuado en niños escolares de Isla de Pascua para conocer el estado de salud bucal y algunos factores de riesgo relacionados con caries dental, por lo tanto, no existe otro registro para realizar una comparación con este trabajo.

Al comparar los resultados obtenidos en este estudio con los observados en el Diagnóstico Nacional de Salud Bucal de Soto y cols. del año $2007^{(9)}$, realizado en Chile continental en niños de 12 años se observa un valor del índice COP-D de 1.9, ligeramente superior al obtenido en este trabajo, 1.19. En este mismo estudio, el porcentaje de niños libres de caries en ambas denticiones es $37.5 \%$, muy inferior al resultado obtenido en este trabajo en piezas permanentes, $61.2 \%$. Extrayendo los resultados observados en el estudio de Soto en la VIII Región, área que al igual que la isla no cuenta con fluoruración del agua de forma natural y/o artificial, se ve una gran diferencia en el índice COP-D, el cual es de 2.09 en esta región a los 12 años comparado con el índice de 1.19 encontrado en este estudio. Por otra parte, el porcentaje de niños libres de caries reportado en la VIII Región es $36.4 \%$, un valor mucho menor al registrado en este trabajo ${ }^{(9)}$

Los resultados de este estudio muestran que el índice COP-D en los escolares del Colegio Católico de Isla de Pascua, oriundos Rapa Nui (COP-D 0.9), es significativamente menor al observado en niños No Rapa Nui (COP-D 1.77) del mismo establecimiento y grupo etáreo. Esta observación podría deberse a los estilos de vida de los niños originarios de la isla. 
Al comparar el Índice Significante de Caries obtenido en este trabajo (SIC 3.33), vemos que es menor al reportado por Cabello y cols., en un estudio efectuado en la Región Metropolitana en niños de 12 años el año 2006, el cual es 3.75 para el estrato socioeconómico alto, 4.28 para el medio y 4.38 para el bajo ${ }^{(19)}$.

A pesar de todas las diferencias descritas para los distintos índices, es difícil efectuar un análisis comparativo entre el índice COP-D registrado, el porcentaje de niños libres de caries y el SIC registrado en niños escolares de Isla de Pascua con niños escolares en el continente debido a que la muestra estudiada en este trabajo se extrajo de un colegio de la isla, y el grupo etáreo era bastante amplio ya que fluctuaba entre 6 y 15 años.

El bajo valor del índice COP-D no podría explicarse utilizando como referencia la cantidad de flúor disponible en el agua, ya que el agua potable de la isla no se encuentra fluorurada artificialmente y en forma natural el nivel de flúor se encuentra bajo el rango preventivo según la especificación Minsal (0.7-1.2 mg/L).

Los menores valores del índice COP-D tampoco pueden ser explicados por la cobertura odontológica puesto que en Isla de Pascua actualmente hay sólo un dentista que atiende de forma regular a nivel público para toda la población, además de operativos ocasionales realizados por distintas instituciones.

El promedio del G-V para el total de niños estudiados es 1.63; lo cual según la categorización de Greene y Vermillon corresponde a un valor clínico de higiene oral de tipo aceptable ${ }^{(13)}$. La frecuencia de las especies bacterianas identificadas en nuestro estudio, mediante la técnica de PCR, fueron de un $56.2 \%$ y $13.2 \%$ para $S$. mutans y $S$. sobrinus, respectivamente. Este resultado no es comparable al obtenido por Salazar y cols. en el año $2008^{(20)}$ en un trabajo realizado en niños residentes de la ciudad de Temuco, quienes determinaron una prevalencia de $88.2 \%$ y $11.8 \%$ para $S$. mutans y S. sobrinus, respectivamente. Es importante destacar que la variabilidad en los resultados podría deberse a diferencias en la sensibilidad del test, la metodología utilizada, la edad y origen de los individuos estudiados, entre otros factores.

Estos resultados nos indican que la frecuencia de patógenos relacionados con caries dental en la isla es menor en relación a una zona del continente que no cuenta con fluoruración del agua, lo cual podría ser un factor que explicaría los mejores valores observados en los índices aplicados.

De acuerdo a la clasificación de riesgo microbiológico al desarrollo de lesiones de caries propuesta por Linossier y cols. ${ }^{(21)}$, según el recuento de S. mutans (3.3×105), se categorizó a esta muestra en riesgo medio de sufrir nuevas lesiones de caries.

En cuanto a la encuesta de dieta, según el análisis efectuado, se observa que el $77 \%$ de los niños escolares presenta riesgo cariogénico bajo-mediano en cuanto a calidad, consistencia y frecuencia de ingesta de alimentos, sin discriminar entre niños Rapa Nui y No Rapa Nui. Este resultado es otro factor que podría explicar los mejores valores en los índices aplicados que presentan los niños escolares de isla de pascua. En un estudio realizado por Macmillan, publicado en el $2005^{(22)}$ sobre hábitos de alimentación en niños de 6 años de Isla de Pascua, se observa concordancia con los resultados obtenidos en este estudio, ya que demostró que la mayor parte de los niños estudiados tienen hábitos alimentarios considerados saludables, que incluyen la ingesta regular de lácteos y frutas tanto en las colaciones escolares como en el hogar y el consumo sólo ocasional de alimentos "chatarra" (completos, pizza, papas fritas, bebidas de fantasía). Además, las colaciones escolares son generalmente traídas desde el hogar e incluyen habitualmente frutas, cereales o lácteos y sólo ocasionalmente galletas, queques o productos similares. En el establecimiento educacional donde fue realizado este estudio no hay kiosco que expenda alimentos al interior del colegio. Cas el total de la muestra consume pescado en su hogar al menos una vez por semana y frutas a diario.

\section{CONCLUSIONES}

Según este estudio con respecto a la dentición permanente, el $38.8 \%$ de los niños de Isla de Pascua tiene historia de caries. El $62 \%$ se encuentra libre de caries.

La media del índice COP-D de la población en estudio es 1.19 En mujeres es 1.2; en hombres, 1.18. En el grupo Rapa Nui es 0.9 y No Rapa Nui, 1.77.

Según el valor del índice COP-D de los niños clasificados como Rapa Nui (0.9), los datos sugieren que este grupo podría presentan mejor estado de salud bucal que los niños catalogados como No Rapa Nui habitantes de Isla de Pascua cuyo valor COP-D es 1.77 .

El estado de Higiene Oral, de acuerdo al índice G-V, de Rapa Nui es 1.69; y de No Rapa Nui, 1.53; de mujeres, 1.60 y hombres, 1.66. Lo cual corresponde a un valor clínico de higiene oral de tipo aceptable para todos los grupos.

El $56 \%$ de los niños Rapa Nui, que fueron examinados microbiológicamente, presentan patógenos bucales relacionados con caries dental.

La dieta para niños escolares de la Isla de Pascua que fueron incluidos en la evaluación de dieta se categoriza como de bajo-mediano riesgo.

El agua potable de Isla de Pascua contiene concentraciones de flúor en forma natural que alcanzan los $0.223 \mathrm{ppm}$.

\section{CONFLICTOS DE INTERÉS}

Los autores declaran no tener conflictos de interés.

\section{REFERENCIAS BIBLIOGRÁFICAS}

1. Fejerskov O, Kidd E. Dental caries: The disease and its clinical management. Second edition, Wiley-Blackwell. 2008.

2. Seppä L, Kärkkäinen S, Hausen H. Caries trends 1992-1998 in two low-fluoride finnish towns formerly with and without fluoridation. Caries Res, 2000; 34: 462-468.

3. Pitts N, Longbottom C. Preventive care adviser (PCA)/Operative care adviser (OCA)-categorising caries by management option. Community Dent Oral Epidemiol, 1995 Feb; 23(1): 55-59.

4. Petersen P. The World Oral Health Report 2003. Continuous improvement of oral health in the 21st century - the approach of the WHO Global Oral Health Program. Community Dent Oral Epidemiol, 2003; 31(suppl.1): 3-24.

5. Petersson HG, Bratthall D. The caries decline: A review of reviews. Eur J Oral Sci, 1996; 104: 436-443.

6. Nadanowsky P, Sheiham A. Relative contribution of dental services to the changes in caries levels of 12-year-old children in 18 industrialized countries in the 1970s and early 1980s. Commun Dent Oral Epidemiol, 1995; 23: 331-339.

7. Konig K. Clinical manifestations and treatment of caries from 1953 to global changes in the 20 century. Caries Res, 2004; 38(3): 168-172.
8. Marthaler T. Changes in dental caries 1953-2003. Caries Res, 2004 38(3): 173-181.

9. Soto L, Tapia R, Jara G, Rodríguez G, Urbina T. Diagnóstico nacional de salud bucal del adolescente de 12 años y evaluación del cumplimiento de los objetivos sanitarios de salud bucal 2000-2010, Chile 2007. Facultad de Odontología, Universidad Mayor. Ediciones Universidad Mayor, 2007. 10. Thylstrup A, Fejerskov O. Textbook of clinical cariology. Second Edition. Munksgaard. 1999.

11. Instituto Nacional de Estadísticas Chile. Estimaciones de población por sexo y edad. Comunas. 1990-2020. Hallado en:

http://www.ine.cl/canales/chile_estadistico/home.php. Acceso el 7 marzo 2009.

12. Encuestas de salud bucodental. Métodos Básicos. Cuarta Edición. Biblioteca Organización Mundial de la Salud, Ginebra, Suiza. 1997.

13. Carranza F, Newman MG, Takei H. Periodontología clínica, Editorial Mc Graw-Hill 9 Edición, 2003: 920 páginas ISBN: 9701042433.

14. Bratthall D. Introducing the Significant Caries Index together with a proposal for a new global oral health goal for 12-year-olds. Int Dent $J$, 2000 Dec; 50(6): 378-384

15. Oho T, Yamashita Y, Shimazaki Y, Kushiyama M, Koga T. Simple and 
rapid detection of Streptococcus mutans and Streptococcus sobrinus in human saliva by polymerase chain reaction. Oral Microbiol Immunol, 2000; 15:258-262.

16. Holm AK, Andersson R. Enamel mineralization disturbances in 12 -year-old children with known early exposure to fluorides. Community Dent Oral Epidemiol, 1982; 10: 335-339.

17. Yévenes I, Zillmann G, Muñoz A, Aranda W, Echeverría S, Hassi $\mathrm{J}$, Espinoza N, Reyes J. Estudio: Impacto de la fluoruración del agua potable en la Región Metropolitana. Ministerio de Salud. Facultad de Odontología. Universidad de Chile. 2005.

18. Yévenes I, Reyes J, Sánchez U, Sanza H. Estudio y determinación de fluoruros, triclosán y citrato de zinc en dentífricos. Av Odontoestomatol, 1999; 15: 433-444.
19. Cabello R, Aranda W, Muñoz A, Gamonal J. Disparities in the distribution of dental caries among adolescent in the Metroplitan Region of Chile. Caries Res, 2008; 42: 202

20. Salazar LA, Vásquez C, Almuna A, Oporto G, Santana R, Herrera $\mathrm{CL}$, Sanhueza A. Detección molecular de estreptococos cariogénicos en saliva. Int J Morphol, 2008; 26(4): 951-958.

21. Linossier A, Vargas A, Zillmann G, Arriagada M, Rojas R, Villegas R. Streptococci mutans: A semiquantitative method to assess the risk to oral infection in preschool Chilean children. Rev Med Chile, 2000; 131 412-418.

22. Macmillan KN. Evaluación del estado nutricional, hábitos de alimentación y actividad física en escolares de $1^{\circ}$ básico de Isla de Pascua. Rev Chil Nutr, 2005; 32(3): 232-237. 